\title{
Sulfur abundance of asteroid 25143 Itokawa observed by X-ray fluorescence spectrometer onboard Hayabusa
}

\author{
Takehiko Arai ${ }^{1}$, Tatsuaki Okada ${ }^{1}$, Yukio Yamamoto ${ }^{1}$, Kazunori Ogawa $^{2}$, Kei Shirai $^{1}$, and Manabu Kato ${ }^{1}$ \\ ${ }^{1}$ Department of Solid Planetary Science, Institute of Space and Astronautical Science, The Japan Aerospace Exploration Agency (ISAS/JAXA), \\ 3-1-1 Yoshinodai, Sagamihara, Kanagawa 229-8510, Japan \\ ${ }^{2}$ Department of Earth and Planetary Sciences, Graduate School of Science and Engineering, Tokyo Institute of Technology, \\ Ookayama, Meguro-ku, Tokyo 152-8551, Japan
}

(Received November 24, 2006; Revised November 25, 2007; Accepted December 2, 2007; Online published February 12, 2008)

\begin{abstract}
The Japanese Hayabusa spacecraft successfully carried out in situ observations of S-class asteroid 25143 Itokawa, including the surface major elemental analysis with the X-ray fluorescence spectrometer (XRSHayabusa). Our previous results for the X-ray experiments (Okada et al., 2006a) indicated that major elemental ratios of $\mathrm{Mg} / \mathrm{Si}$ and $\mathrm{Al} / \mathrm{Si}$ on the surface of Itokawa resemble ordinary LL- or L-chondrites more than any other meteorite analogues. In the NEAR Shoemaker observations of S-class asteroid 433 Eros, the results of X-ray fluorescence observations indicated the depletion of sulfur, probably reflecting impact-induced volatilization, photoor ion-induced sputtering at the surface, or the loss of FeS-rich materials due to partial melting. Here, we determined the elemental abundance of sulfur $(\mathrm{S})$ on the surface of Itokawa, in addition to that of $\mathrm{Mg}, \mathrm{Al}$, and $\mathrm{Si}$, and its regional variation using XRS-Hayabusa observations. In particular, we carefully corrected the fluctuation of solar X-rays, variation of surface geometry, and sensor response function in this analysis, and thus we believe that the results are more accurate than those of our previous report. In this study, the upper and lower limits for $\mathrm{Mg} / \mathrm{Si}$, $\mathrm{Al} / \mathrm{Si}$, and $\mathrm{S} / \mathrm{Si}$ overlap those of meteorite analogues for ordinary chondrites or primitive achondrites. In terms of the major elemental composition, Itokawa is best classified as a ordinary chondrite or a primitive achondrite. Our models do not include the mineral mixing effects. With the effects, the abundance of sulfur is expected to be $30 \%$ lower than our results. Hence, we conclude that the abundance of sulfur on the surface of Itokawa is almost equal to or even lower than the average abundance in ordinary chondrites. Although the abundances for $\mathrm{Mg}$ and $\mathrm{Si}$ are globally homogeneous, best-fit or upper limits of mass fraction for $\mathrm{Al}$ and $\mathrm{S}$ vary in local areas. There is a negative correlation $(-0.92)$ for $\mathrm{Al} / \mathrm{Si}$ vs. $\mathrm{S} / \mathrm{Si}$ in ten facets. In particular, the area with the lowest sulfur, accompanied with enriched aluminum, is found in Arcoona, close to a cratered area. Therefore, aluminum enrichment and sulfur depletion features may support events of partial melting on the parent body of Itokawa or aluminum-rich material impacts on the surface of Itokawa. In some areas, Itokawa has a brighter geometric albedo and color variation. Little altered, fresh material may be exposed in these portions of the surface. The sulfur abundance on the surface appears to vary between little and highly altered areas by space weathering. Thus, the sulfur regional variation in our result may reflect the heterogeneity of a surface altered by space weathering.
\end{abstract}

Key words: Hayabusa, XRS, elemental composition, abundance, Itokawa, sulfur, space weathering.

\section{Introduction}

The Japanese Hayabusa spacecraft successfully carried out in situ observations of asteroid 25143 Itokawa (Fujiwara et al., 2006). The X-ray fluorescence spectrometer onboard Hayabusa (XRS-Hayabusa) observed X-ray emission from the surface of Itokawa which determined the elemental composition. Itokawa is classified as an S-class, meaning a stony asteroid, and the spectral feature is similar to ordinary chondrites, especially to LL-chondrites (Binzel et al., 2001). The primary goal of XRS-Hayabusa is to determine a relationship between S-class asteroid Itokawa and known meteorite analogues classified in laboratory measurements, including a fundamental question of whether the asteroid is a parent body of meteorites or not. Our previous result

Copyright (c) The Society of Geomagnetism and Earth, Planetary and Space Sciences (SGEPSS); The Seismological Society of Japan; The Volcanological Society of Japan; The Geodetic Society of Japan; The Japanese Society for Planetary Sciences; TERRAPUB.
(Okada et al., 2006a) has estimated that the major elemental composition ratios for $\mathrm{Mg} / \mathrm{Si}$ and $\mathrm{Al} / \mathrm{Si}$ on the surface of Itokawa resemble ordinary LL- or L-chondrites more than any other meteorites analogues. Also, results of Near Infrared Spectrometer onboard Hayabusa (NIRS-Hayabusa) have indicated that olivine/pyroxene composition ratios derived from about $1-\mu \mathrm{m}$ absorption band are most similar to LL-chondrites (Abe et al., 2006). These results imply that the S-class asteroid Itokawa is a parent body of ordinary chondrites.

Fluorescent X-rays are excited on an asteroid surface by solar X-rays. In observing solid planetary bodies, XRSHayabusa can detect X-ray fluorescence line spectra of $\mathrm{Mg}$, $\mathrm{Al}$, and $\mathrm{Si}$ even in quiescent solar conditions, and it can also detect other elements such as $\mathrm{S}, \mathrm{Ca}$, and $\mathrm{Fe}$ in flared solar conditions. Since the detectable depth for the fluorescent X-rays is within the depth of $100 \mu \mathrm{m}$, XRS-Hayabusa can determine the major elements on the uppermost surface. 

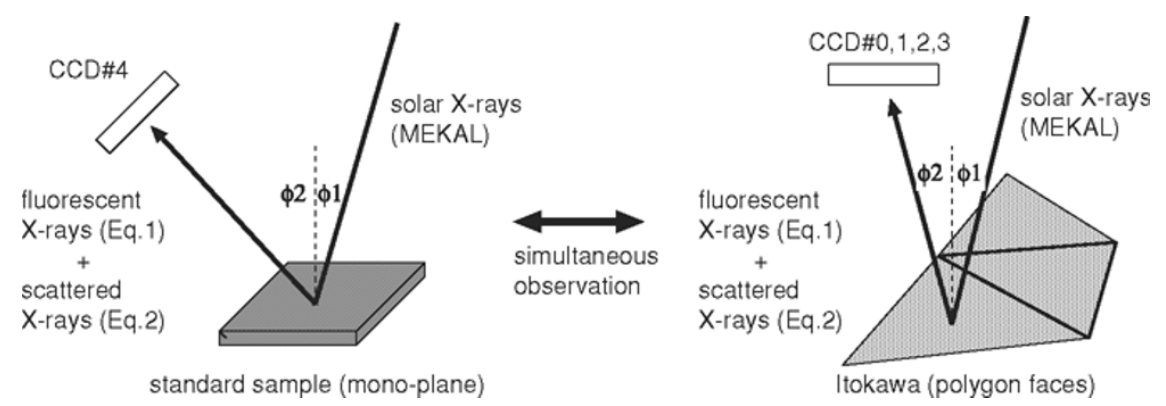

Fig. 1. Schematic views of the observation for standard sample and Itokawa (words in parentheses indicate X-ray models). The standard sample and Itokawa observation are simultaneously performed in all observations. The CCD\#4 observes the X-rays from a standard sample excited by incident solar X-rays. CCD\#0, 1, 2, and 3 observe X-rays from the surface of Itokawa excited by the same incident solar X-rays. The geometry of observation area on the surface of Itokawa varies in local areas. Therefore, we calculate the incident and emission X-ray angles for the surface with shape model of Itokawa (Demura et al., 2006).

Here, we focus our attention on the abundance of sulfur (S) on the surface of Itokawa. Since sulfur is highly volatile element, we can obtain a redox condition of asteroid formation points or degree of fractionation due to partial melting from the abundance of sulfur. Additionally, the abundance of sulfur on the surface of the asteroid will be modified by some processes, such as meteoritic impacts or a space weathering.

Based on X-ray fluorescence observation of asteroid 433 Eros with X-ray spectrometer onboard the Near-Earth Asteroid Rendezvous (NEAR)-Shoemaker, Trombka et al. (2000) and Nittler et al. (2001) reported that the ratio for $\mathrm{S} / \mathrm{Si}$ on the surface is much lower than that of ordinary chondrites. Nittler et al. (2001) suggested that the reason for low $\mathrm{S} / \mathrm{Si}$ is impact-induced volatilization and/or photoor ion-induced sputtering at the surface of the asteroid, or the loss of FeS-rich material due to partial melting. Also, a sulfur volatilization process due to space weathering, such as micro-meteorite impacts or/and energetic erosion due to solar wind sputtering, has been proposed by Killen (2003) and Kracher and Sears (2005). Their timescale estimation for sulfur depletion in the uppermost layer of 10-100 $\mu \mathrm{m}$ due to space weathering is the order of $10^{6}$ years, so that sulfur on the surface can easily be taken away from the uppermost layer. Thus, sulfur abundance on the asteroid surface will indicate not only the physical conditions of asteroid formation points but also the history of surface modification.

In the observations of Itokawa, brightness and color variation on the surface have been found with the multiband imager AMICA-Hayabusa (e.g., Ishiguro et al., 2006; Sasaki et al., 2006) and the near-infrared spectrometer NIRS-Hayabusa (Abe et al., 2006). These heterogeneities might be caused by space weathering and, if so, sulfur abundance might have been affected by that process. Therefore, it is significantly important that we determine the sulfur abundance and its regional variation with XRS-Hayabusa observations and discuss the possible sulfur modification process that must have occurred on the surface of Itokawa.

\section{XRS-Hayabusa}

$\mathrm{XRS}$-Hayabusa is an X-ray fluorescence spectrometer based on a charge-coupled device (CCD) that has a good energy resolution (Okada et al., 2000, 2006b) relative to the past X-ray detectors of planetary missions, such as Apollo 15/16 and NEAR-Shoemaker. The effective area of XRS is $25 \mathrm{~cm}^{2}(4 \times \mathrm{CCD})$, and effective pixels of CCD are $1024 \times 1024$, with a pixel size being $24 \mu \mathrm{m}^{2}$. The CCD is used not for imaging but photon counting of X-rays. The asteroid detector, with an array of four CCDs (CCD\#0, 1, 2,3 ), observes X-ray photons from the surface of the asteroid, while another single CCD (CCD\#4) observes X-rays from an onboard standard sample, which is used for a comparative analysis or indirectly monitoring the solar X-rays. Energy resolution (full width at half maximum) of CCDs is about $200 \mathrm{eV}$ in the range of 0.7 to $10.0 \mathrm{keV}$. The footprint as observation area is limited with a mechanical collimator whose field of view is $3.5^{\circ} \times 3.5^{\circ}$. Bright visible reflection from the asteroid is shielded with a beryllium window of $5 \mu \mathrm{m}$ thickness.

The CCD\#4 (solar monitor) observes X-ray emission from the standard sample excited by solar X-rays, and the CCD\#0, 1, 2, 3 (asteroid detector) simultaneously observes $\mathrm{X}$-ray emission from an asteroid excited by solar X-rays, as shown schematically in Fig. 1. Since the solar X-rays fluctuate all the time, simultaneous observations of both the standard sample and the asteroid need to be obtained. The composition of the standard sample is known (Table 1), and thus the solar monitor can indirectly estimate spectral intensities and the profile of solar X-rays. When the solar monitor estimates the solar X-rays as the X-ray exciting source, the asteroid detector quantitatively determines the elemental composition of the asteroid because generated fluorescent X-rays are proportional to solar X-rays.

\section{Observation of Itokawa}

During September to November in 2005, XRS-Hayabusa observed the surface of Itokawa from a distance of 20 to $0 \mathrm{~km}$. Unfortunately, two reaction wheels controlling the attitude of Hayabusa were broken in late July and early October, and XRS-Hayabusa could not observe for a long time continuously in the direction of Itokawa. Fortunately, some observation data were sufficient to determine the elemental composition on Itokawa when solar flares occurred. In particular, solar activity was high enough for X-ray spectrometry in the first-touchdown and away (TD\#1) operation, obtaining a sample collection on 19 November 2005. 
Table 1. Elemental abundances of standard sample and average abundances of ordinary-chondrites (wt.\%).

\begin{tabular}{ccrrr}
\hline Element & Standard sample & \multicolumn{1}{c}{$\mathrm{H}(229)$} & \multicolumn{1}{c}{$\mathrm{L}(256)$} & \multicolumn{1}{c}{ LL (83) } \\
\hline $\mathrm{H}$ & - & $0.11 \pm 0.09$ & $0.04 \pm 0.07$ & $0.06 \pm 0.11$ \\
$\mathrm{C}$ & - & $0.03 \pm 0.08$ & $0.07 \pm 0.19$ & $0.04 \pm 0.11$ \\
$\mathrm{O}$ & 40.80 & $34.51 \pm 1.68$ & $36.96 \pm 1.02$ & $38.49 \pm 1.27$ \\
$\mathrm{Na}$ & 2.30 & $0.56 \pm 0.08$ & $0.66 \pm 0.08$ & $0.69 \pm 0.08$ \\
$\mathrm{Mg}$ & 8.70 & $14.13 \pm 0.51$ & $15.11 \pm 0.42$ & $15.39 \pm 0.59$ \\
$\mathrm{Al}$ & 5.20 & $1.19 \pm 0.23$ & $1.26 \pm 0.20$ & $1.37 \pm 0.35$ \\
$\mathrm{Si}$ & 19.70 & $16.55 \pm 0.60$ & $18.21 \pm 0.49$ & $18.58 \pm 0.89$ \\
$\mathrm{P}$ & - & $0.11 \pm 0.05$ & $0.12 \pm 0.04$ & $0.12 \pm 0.05$ \\
$\mathrm{~S}$ & $<1.00$ & $2.10 \pm 0.43$ & $2.24 \pm 0.41$ & $2.10 \pm 0.57$ \\
$\mathrm{~K}$ & 0.35 & $0.07 \pm 0.02$ & $0.08 \pm 0.02$ & $0.09 \pm 0.02$ \\
$\mathrm{Ca}$ & 5.75 & $1.17 \pm 0.14$ & $1.29 \pm 0.11$ & $1.34 \pm 0.15$ \\
$\mathrm{Ti}$ & 0.60 & $0.05 \pm 0.02$ & $0.06 \pm 0.02$ & $0.07 \pm 0.02$ \\
$\mathrm{Cr}$ & 0.35 & $0.30 \pm 0.23$ & $0.34 \pm 0.24$ & $0.33 \pm 0.07$ \\
$\mathrm{Mn}$ & 0.35 & $0.22 \pm 0.05$ & $0.25 \pm 0.04$ & $0.25 \pm 0.04$ \\
$\mathrm{Fe}$ & 15.00 & $27.28 \pm 1.96$ & $22.07 \pm 1.28$ & $20.12 \pm 1.79$ \\
$\mathrm{Co}$ & 0.35 & $0.06 \pm 0.02$ & $0.05 \pm 0.02$ & $0.03 \pm 0.02$ \\
$\mathrm{Ni}$ & 0.70 & $1.52 \pm 0.23$ & $1.15 \pm 0.15$ & $0.93 \pm 0.27$ \\
\hline total & 100.15 & 99.96 & 99.96 & 100.00
\end{tabular}

Table 2. Observation status of standard sample and Itokawa.

\begin{tabular}{|c|c|c|c|c|c|c|c|c|}
\hline & \multicolumn{3}{|c|}{ Standard sample } & \multicolumn{5}{|c|}{ Itokawa } \\
\hline $\begin{array}{l}\text { Observation } \\
\text { start time } \\
\text { (UTC) }\end{array}$ & $\begin{array}{l}\text { Area } \\
\left(\mathrm{cm}^{2}\right)\end{array}$ & $\begin{array}{c}\text { Incident } \\
\text { angle } \phi_{1} \\
\text { (deg) }\end{array}$ & $\begin{array}{c}\text { Emission } \\
\text { angle } \phi_{2} \\
(\mathrm{deg})\end{array}$ & $\begin{array}{c}\text { Facet } \\
\text { number }\end{array}$ & $\begin{array}{l}\text { lon. } \\
\text { (deg) }\end{array}$ & $\begin{array}{l}\text { Area } \\
\left(\mathrm{m}^{2}\right)\end{array}$ & $\begin{array}{c}\text { Incident } \\
\text { angle } \phi_{1} \\
\text { (deg) }\end{array}$ & $\begin{array}{l}\text { Emission } \\
\text { angle } \phi_{2} \\
(\mathrm{deg})\end{array}$ \\
\hline $12: 02: 11$ & & 0.0 & & 1 & 306.3 & 20386 & 31.0 & -29.5 \\
\hline $12: 39: 52$ & & 0.0 & & 2 & 296.0 & 18683 & 27.6 & -27.5 \\
\hline $13: 17: 30$ & & -0.2 & & 3 & 285.3 & 17939 & 27.2 & -29.8 \\
\hline $13: 55: 12$ & & -7.4 & & 4 & 272.5 & 17962 & 30.8 & -36.6 \\
\hline $14: 32: 53$ & 14.76 & 0.1 & -45.0 & 5 & 259.9 & 16408 & 42.2 & -49.1 \\
\hline $15: 10: 37$ & & -0.2 & & 6 & 251.6 & 13985 & 56.0 & -62.6 \\
\hline $15: 48: 20$ & & 2.6 & & 7 & 237.6 & 9595 & 67.2 & -73.8 \\
\hline $16: 26: 04$ & & 0.1 & & 8 & 221.3 & 8606 & 46.6 & -53.3 \\
\hline $17: 03: 48$ & & -2.7 & & 9 & 184.8 & 7429 & 21.3 & -25.4 \\
\hline $17: 41: 32$ & & 0.1 & & 10 & 168.9 & 6111 & 43.1 & -36.2 \\
\hline
\end{tabular}

During the early time of TD\#1 phase, the solar flare events were observed by the solar observation satellites GOES 10 and 12 (GOES's web site), and then the solar monitor of XRS-Hayabusa also sensed solar flare events. This indicates that solar activity was relatively enhanced at Itokawa, the opposite side of the Earth from the Sun.

Hayabusa descended along the equator of Itokawa from west to east together with the rotation of Itokawa (12 hperiod rotation). Although XRS-Hayabusa observations were continuously carried out, we used the observed data obtained at a distance of 0.94 to $0.38 \mathrm{~km}$, where observed surface areas of Itokawa varied between 20,386 and $6,111 \mathrm{~m}^{2}$. The off-normal angle of the Sun to the Standard sample was less than $10^{\circ}$, and the off-normal angle of CCD\#4 to the Standard sample was fixed $45^{\circ}$. Also, the angle of the Sun-to-Itokawa-to-XRS-Hayabusa was less than $10^{\circ}$, but the incident and emission X-ray angles were varied due to the surface geometry for the observed planes between $0^{\circ}$ and $90^{\circ}$. Table 2 shows the observation start time, observed area, average incident, and emission angles for ten observation areas called a facet.

The CCDs were cooled with a peltier cooler, and so these temperatures were stable at $-52 \pm 1{ }^{\circ} \mathrm{C}$ during this period.
This temperature enables good energy resolution, but the data during landing on Itokawa are noisy due to an increase in the fluctuation of dark current accompanied by an increasing temperature of the CCDs. Therefore, we analyzed only the data obtained during the Hayabusa decent phase, including the data of our previous report (Okada et al., 2006a).

The observation data are discontinuous because the onboard computer of XRS-Hayabusa controlled the data-rate in order to reduce the data, and the observation data are spectra, integrated every $300 \mathrm{~s}$. Also, there are background update time of $60 \mathrm{~s}$ as a dead-time per integration intervals (Yamamoto, 2002).

\section{X-ray Models}

XRS-Hayabusa observes both fluorescent and scattered $\mathrm{X}$-rays from the surface of Itokawa and the onboard standard sample, excited by incident solar X-rays. The intensity of fluorescent and scattered X-rays depends on model parameters, such as emission-measures as plasma density and size, and plasma temperature of the solar coronal region. Fluorescent $\mathrm{X}$-rays are line spectra whose intensities are proportional to elemental abundance. Scattered X-rays are 
(a) Solar X-rays

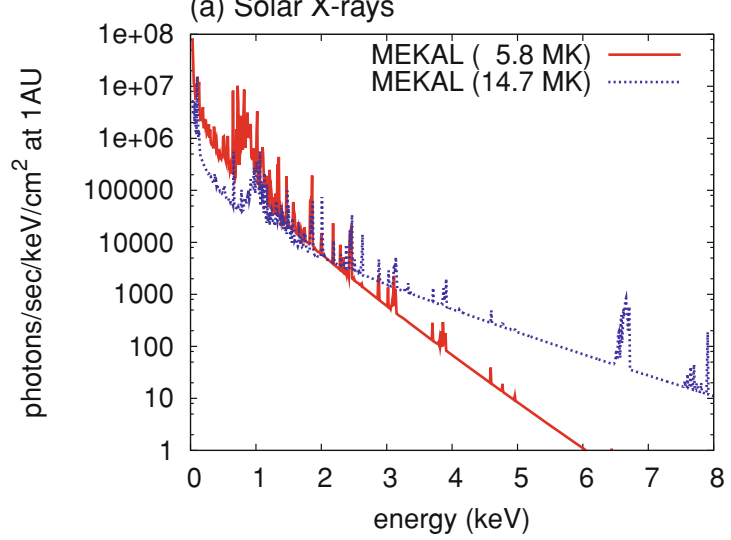

(b) X-rays from Standard sample

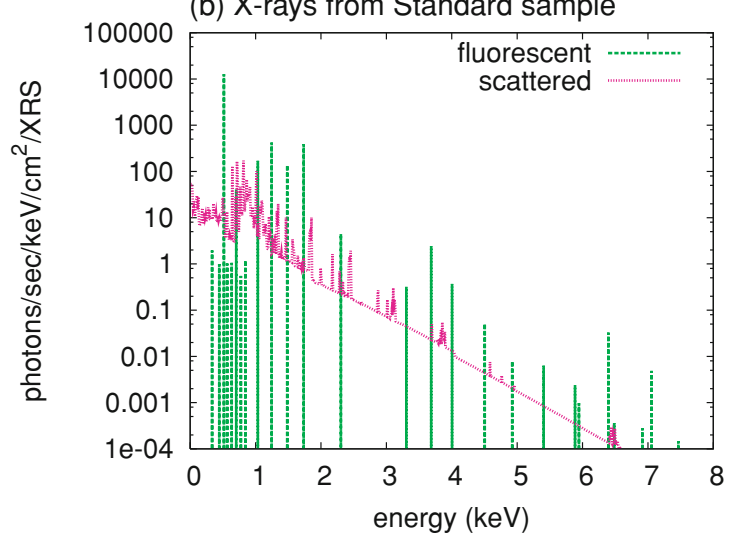

(c) X-rays from Ordinary chondrites

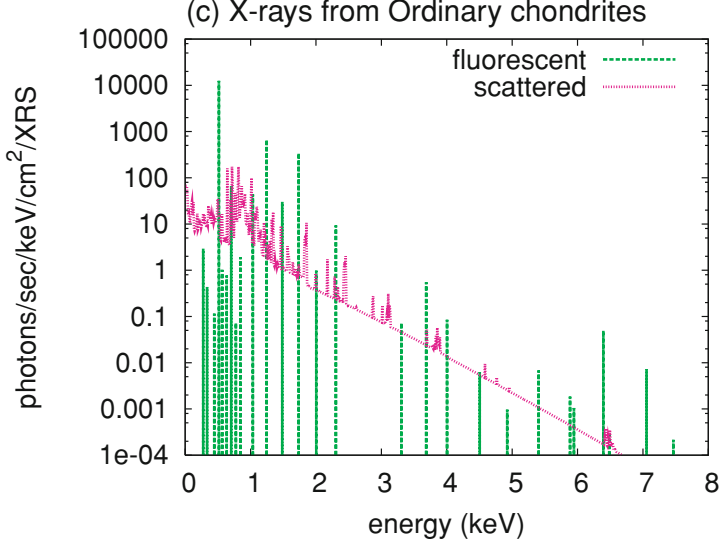

Fig. 2. Examples of the calculated X-ray spectral models are shown. (a) The example spectra of MEKAL model. The blend spectrum of MEKAL models fits to observation spectrum of standard sample in the range of soft and hard energy. (b) The example model spectra of $\mathrm{X}$-ray emission from standard sample. It contains the line spectra of fluorescent $\mathrm{X}$-rays and continuum spectrum of scattered X-rays. Major peaks are $\mathrm{O}-\mathrm{K} \alpha(0.52 \mathrm{keV}), \mathrm{Mg}-\mathrm{K} \alpha(1.25 \mathrm{keV}), \mathrm{Al}-\mathrm{K} \alpha(1.49 \mathrm{keV})$, $\mathrm{Si}-\mathrm{K} \alpha(1.74 \mathrm{keV}), \mathrm{S}-\mathrm{K} \alpha(2.31 \mathrm{keV}), \mathrm{Ca}-\mathrm{K} \alpha / \beta$ (3.69 keV / $4.01 \mathrm{keV})$, and $\mathrm{Fe}-\mathrm{K} \alpha / \beta(6.40 \mathrm{keV} / 7.06 \mathrm{keV})$. (c) The example model spectra of $\mathrm{X}$-ray emission from the analogues of ordinary chondrites. Same energy lines as the X-rays from standard sample are found in this figure.

coherent and incoherent scatter of solar X-rays, composed of characteristic lines of plasma and continuum spectrum as thermal bremsstrahlung. In order to determine the elemental abundance, we need to quantitatively estimate the fluorescent X-rays and to separate the fluorescent X-rays and the scattered X-rays from observation spectrum. Hence, we make precise X-ray models, such as solar X-rays, fluores- cent X-rays, and scattered X-rays.

\subsection{Solar X-rays}

The model of a solar X-ray spectrum $J$ as function of energy is described as thermal bremsstrahlung modeled by Mewe et al. (1985) as the Mewe-Kaastra-Liedahl (MEKAL) model (see also Arnaud et al., 2005). The variables in the model of the solar X-ray are the emissionmeasures as plasma density and size, and the temperature of the solar coronal region where the solar X-rays are emitted. Solar abundance is assumed to be in line with values from Anders and Grevesse (1989). Two examples of MEKAL model spectra with different emission-measure and temperature are shown in Fig. 2(a), where the spectral intensity is proportional to emission-measure and the spectral shape becomes harder for increasing temperature.

\subsection{Fluorescent X-rays}

The model of a fluorescent X-ray spectrum $I^{f}$ as a function of fluorescent X-ray energy $E_{i}^{f}$ for element $i$ (e.g., Bearden, 1967) is described as discrete lines which represent characteristic X-rays. These intensities are proportional to elemental abundance as mass fraction $w_{i}$ for element $i$. The fluorescent $\mathrm{X}$-ray is generated when the energy of the solar X-ray is higher than the X-ray absorption edge energy $E_{i}^{\text {edge }}$ for element $i$. The incident solar X-rays are attenuated exponentially like an electromagnetic wave in the ratio of $\mu_{i}$ for element $i$ as a function of energy called attenuation coefficient (e.g., Chantler et al., 2005) in the surface layer, and then the X-rays are absorbed in the ratio of $\tau_{i}$ for element $i$ as function of energy called absorption coefficient (e.g., Chantler et al., 2005) in the near layer. The portion of absorbed X-rays are converted to fluorescent $\mathrm{X}$ rays in the ratio of $\omega_{i}$ for element $i$ called fluorescent yield (e.g., Krause, 1979), which is K or L line emission yield of fluorescent X-ray. The intensities of fluorescent X-ray are written as follows (e.g., Jenkins et al., 1995; Clark and Trombka, 1997),

$$
I^{f}\left(E_{i}^{f}\right)=\frac{\Omega S}{4 \pi} \int \frac{\omega_{i} p_{i} j_{i} t_{i} w_{i} \tau_{i}(E) J(E)}{\mu_{j}(E)+\mu_{j}\left(E_{i}^{f}\right) \frac{\cos \phi_{1}}{\cos \phi_{2}}} d E,
$$

where $\Omega$ is the field of view of XRS-Hayabusa (observation area per squares of altitude), $S$ is effective area of XRSHayabusa (squares of 1 inch per CCD), $p_{i}$ is the ratio of $\alpha$ or $\beta$ line emission for element $i$ assuming approximately the ratio of $\alpha: \beta=15: 2), j_{i}$ is the jump ratio which is $\mathrm{K}$ or $\mathrm{L}$ shell absorption ratio for element $i$ (Chantler et al., 2005), $t_{i}$ is the ratio of shell transition probability for element $i$ $(\sim 1), \phi_{1}$ and $\phi_{2}$ are incident solar X-rays and emission fluorescence X-ray angles, respectively (see Fig. 1). The range of integration energy is from edge energy (Chantler et al., $2005)$ to infinity. The model examples of X-ray emission from standard sample and ordinary chondrites analogues are shown in Fig. 2(b) and (c), respectively, where the green lines show the model spectra of fluorescent X-rays.

\subsection{Scattered X-ray}

The model of a scattered X-ray spectrum as continuum emission $I^{s}$ for element $i$ is composed of coherent Rayleigh scatter and incoherent Compton scatter. Here, Compton scatter is smaller than Rayleigh scatter in the degree of order in the range of less than $10 \mathrm{keV}$, and thus Compton scatter 
is neglected. Rayleigh scatter of solar X-ray is written as (e.g., Thomson, 1906; Clark and Trombka, 1997),

$$
I^{s}\left(E_{i}\right)=\Omega S \frac{w_{i}\left(\frac{N_{A}}{M_{i}} r_{e}^{2} \frac{1+\cos ^{2} \theta}{2} f_{i}^{2}\right) J\left(E_{i}\right)}{\mu_{i}\left(E_{i}\right)\left(1+\frac{\cos \phi_{1}}{\cos \phi_{2}}\right)},
$$

where $N_{A}$ is the Avogadro constant of $6.02 \times 10^{23} \mathrm{~mol}^{-1}$, $r_{e}$ is the classical electron radius of $2.82 \times 10^{-13} \mathrm{~cm}, \theta$ is a back scatter angle of $\theta=180^{\circ}-\left(\phi_{1}+\phi_{2}\right), M_{i}$ is atomic weight for element $i$ (Chantler et al., 2005), and $f_{i}$ is the atomic scattering factor for element $i$ (Maslen et al., 2004) as a function of scatter angle. The model examples of $\mathrm{X}$-ray emission from standard sample and ordinary chondrites analogues are shown in Fig. 2(b) and (c), respectively, where the pink lines show the model spectra of scattered Xrays.

\subsection{Matrix effect}

When X-rays are attenuated in objects, attenuation ratios are varied for each element $i$ of objects in the ratios of mass fraction $w_{i}$. Thus, we are required to correct this effect called matrix effect, where Eqs. (1) and (2) of attenuation coefficient $\mu_{i}(E)$ are rewritten with the mass fraction $w_{i}$ as follows,

$$
\mu_{i}(E) \rightarrow \sum_{j} w_{j} \mu_{j}(E)
$$

\section{Analysis of Observed Spectra}

Our previous report (Okada et al., 2006a) was based on a comparative analysis (Masuda, 2002) for TD\#1 data, and directly compared the intensities of observed X-ray line spectra off the surface of Itokawa with those off the standard sample. We estimated the line X-ray intensities characteristic of $\mathrm{Mg}, \mathrm{Al}$, and $\mathrm{Si}$, where a polynomial function is used for the continuum spectrum and a Gaussian function is assumed as the response for each line spectrum. The phase angle (the Sun-to-Itokawa-to-detector) was fixed at $0^{\circ}$ since the attitude of spacecraft was controlled to keep the phase angle within $0^{\circ}$ to $10^{\circ}$ during the descent. Although these simple assumptions are practical and often used for X-ray spectroscopy, there are potential problems. (1) Using a polynomial function as spectral background is not always the most practical way for quantitative analysis, when we are able to construct precise background functions, such as scattered solar X-rays, space radiation, or instrumental background. One of the observed spectral backgrounds is scattered solar X-rays, which can be modeled along with the original solar X-rays by analyzing the observed X-ray spectra off the standard sample. Additionally, we assume two spectral backgrounds. One is a constant spectrum in the whole energy range due to space radiation of high-energy particles (see constant spectrum as black line of Fig. 4). High-energy events disperse in many pixels of $\mathrm{CCD}$, and these empirically make a constant spectrum as has been estimated by X-ray astronomical satellite, ASCA. In our observations, anticipated intensities of high-energy events are about an order of magnitude larger than those of ASCA night earth observation, $\sim 0.001$ counts $/ \mathrm{sec} / \mathrm{keV} / \mathrm{cm}^{2}$ (HEASARC's web site), because space radiation is large in interplanetary conditions relative to the Earth's orbit. The other is an exponential spectrum due to outer events of the hot-pixels that happen to make fake events due to charge intrusion from electrode of CCD. The function of onboard software much reduced hot-pixels, but the outer value of hot-pixels still remains and makes exponential spectrum (see exponential spectrum as black line of Fig. 4). (2) Temporal change of the observed phase angle causes apparent changes in the elemental composition ratio, which needs to be corrected using a precise surface geometry. In addition, $\mathrm{X}$-ray incident and emission angles also affect the spectral shape, so that we calculated those angles at each part of the asteroid surface by using the shape model of Itokawa (Demura et al., 2006). (3) The actual response function of XRS-Hayabusa is not a Gaussian but a Gaussian-like function. Therefore, a precise response function needs to be constructed. Since the response function of XRS-Hayabusa has been measured in the pre-launch tests (Arai, 2003), we use it in this study. This response function will be published on Hayabusa's web site as the response matrix for XRS-Hayabusa.

Sulfur abundance of the standard sample is almost equal to zero because it was vaporized in the manufacturing process (see Table 1), and thus we can not use the comparative analysis for sulfur. Therefore, we did not use the standard sample for comparative analysis but the solar X-ray monitor instead, and then we estimate the solar X-ray spectra.

The integration time was $1800 \mathrm{~s}$ for one spectrum in order to obtain statically significant counts of X-ray photons, which needs to acquire at least over 100 photons to achieve within $10 \%$ errors of counting statistics (Knoll, 2000).

We used the least squares fit for comparing the observed and modeled spectra. The fitting algorithms were the simplex method (Press et al., 2002a) and Levenberg-Marquardt method (Press et al., 2002b). The simplex method performs well for fit, but error cannot be estimated. Therefore, we used the simplex method for the initial fit and determined the standard error using the Levenberg-Marquardt method. 5.1 Estimation of Solar X-rays

In order to estimate solar X-rays, we performed a modelfit using the fitting function of fluorescent and scattered $\mathrm{X}$ ray of Eqs. (1) and (2), respectively, and then these functions are convolved with the response function. Since solar activity varied time to time, one MEKAL model could not fit to the observed spectrum in the all-energy range. Thus, we used the blend model of two MEKAL models as the soft-energy spectrum $J_{1}(E)$ and hard-energy spectrum $J_{2}(E)$; solar X-ray spectrum $J$ is then rewritten as $J=n_{1} J_{1}+n_{2} J_{2}$, where $n_{1}$ and $n_{2}$ are proportionality constant used as a free parameter for fit.

Elemental composition of the standard sample was fixed in this calculation, as shown in Table 1. The incident angle $\phi_{1}$ and emission angle $\phi_{2}$ of Eqs. (1) and (2) are shown in Table 2 (average angles for 1800-s observation are only indicated). Free parameters are $n_{1}, n_{2}$, solar coronal temperatures of the MEKAL models, and standard deviation of Gaussian-like response function. Also, free parameters of the constant and exponential backgrounds are the only proportional constants.

Since the standard sample is a glass plate which consists 


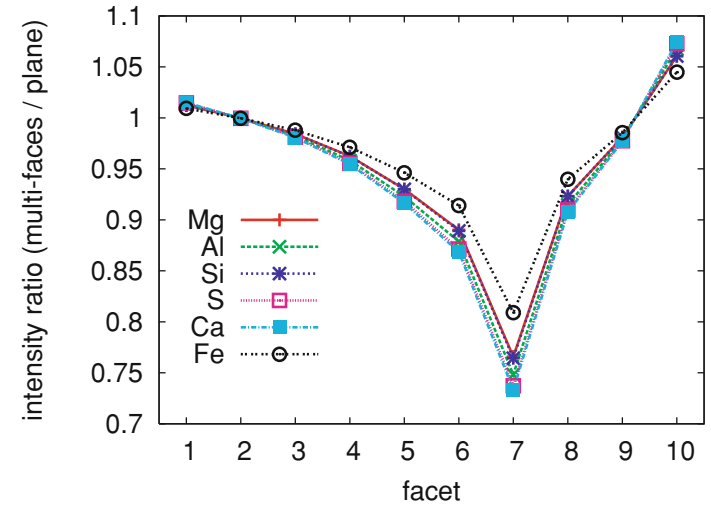

Fig. 3. Intensity ratios of multi-faces to mono-plane for fluorescent lines of $\mathrm{Mg}, \mathrm{Al}, \mathrm{Si}, \mathrm{S}, \mathrm{Ca}$, and $\mathrm{Fe}$ in ten facets. Fluorescent X-rays from multi-faces are calculated with shape model of Itokawa (Demura et al., 2006), varied with incident and emission X-rays angles. Fluorescent $\mathrm{X}$-rays from mono-plane are calculated in same area as multi-faces fixed with incident and emission angles $\left(0^{\circ}\right)$. In particular, the ratio of $\mathrm{Fe}$ varies largely relative to that of other elements. Since most sulfur is composed in Troilite (FeS), the correction of geometry is important for the sulfur estimation.

of a homogeneous blend of elements, apparent changes of $\mathrm{X}$-ray intensities due to surface roughness or mineral composition are negligible.

The example of a best-fit X-ray model to the observed spectrum of standard sample is shown in Fig. 4(a). We found peaks of $\mathrm{Mg}-\mathrm{K} \alpha(1.25 \mathrm{keV})$ and $\mathrm{Si}-\mathrm{K} \alpha(1.74 \mathrm{keV})$, but that of Al-K $\alpha(1.49 \mathrm{keV})$ and $\mathrm{S}-\mathrm{K} \alpha(2.31 \mathrm{keV})$ were not found due to a lower composition in standard sample. Also, an unexplained peak was found in the energy of $2.1 \mathrm{keV}$. This peak is near to the $\mathrm{P}-\mathrm{K} \alpha(2.01 \mathrm{keV})$ line or $\mathrm{Au}-\mathrm{M} \alpha$ $(2.12 \mathrm{keV})$ line. The abundance of $\mathrm{P}$ on the standard sample is too low to be detected (see Table 1) in this solar condition or this integration time. Although $\mathrm{Au}$ is not present in the standard sample, it is used for a meshed support frame of beryllium X-ray window (the optical shield). Since we have not yet determined yet whether the origin of the peak is $\mathrm{Au}$ or instrumental noise, we neglected it in this study.

\subsection{Estimation of elemental composition of Itokawa}

The model of fluorescent and scattered X-rays from the surface of Itokawa is calculated with basically the same method as the estimation of solar X-rays. Since the solar coronal temperatures could derive the best-fit results of solar X-ray estimation, an undetermined parameter for this fit is the elemental composition of Itokawa.

The surface geometry on Itokawa varied in observed areas. The X-ray incident and emission angles at each area are required for analysis by using the precise asteroid shape. We calculated X-ray emissions with the shape model of Aizu 5.02 (Demura et al., 2006) in sunlit areas, and then we corrected the X-ray incident angle $\phi_{1}$ and emission angle $\phi_{2}$ of Eqs. (1) and (2) for the normal vectors of polygon faces within the observed area. Also, we calculated the field of view, and then we rewrote $\Omega$ of the Eqs. (1) and (2) for polygon faces as follows,

$$
\Omega \rightarrow \sum_{i} \frac{s_{i}}{d_{i}^{2}}
$$

where $s_{i}$ is an area for polygon face $i$ and $d_{i}$ is a distance (a) Observed and best-fit spectra of standard sample

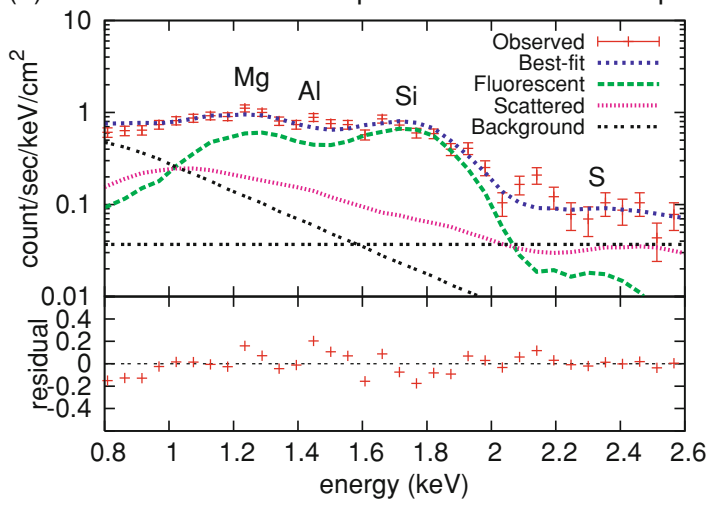

(b) Observed and best-fit spectra of Itokawa

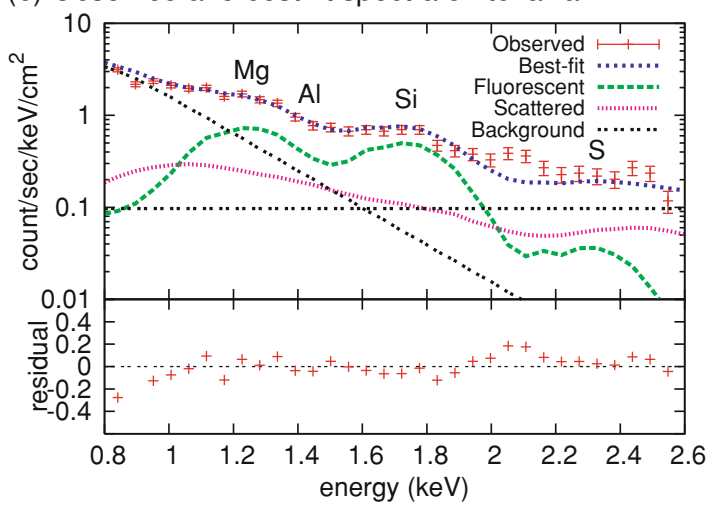

Fig. 4. The observed X-ray spectra of the standard sample and Itokawa are shown together with the fitted spectra. (a) The example of best-fit X-ray model spectra to observed spectrum of standard sample (facet \#10). Red points indicate the observed spectrum with counting statistic errors. Blue line indicates the best-fit spectrum. Green line indicates only fluorescent X-ray model spectrum, and pink line indicates only scattered $\mathrm{X}$-rays model spectrum. Black lines indicate the background spectra of instrument and space radiation. All the model functions are convolved Gaussian-like response of XRS-Hayabusa. Fitted peaks are found in the energy of $\mathrm{Mg}-\mathrm{K} \alpha(1.25 \mathrm{keV}), \mathrm{Si}-\mathrm{K} \alpha(1.74 \mathrm{keV})$, but peaks of Al-K $\alpha$ and $(1.49 \mathrm{keV}) \mathrm{S}-\mathrm{K} \alpha(2.31 \mathrm{keV})$ are not found due to faintness. Also, an unexplained peak is found in the energy of about $2.1 \mathrm{keV}$. In this period, the best-fit model to the solar plasma temperatures for observed spectra are 13.5-15.7 MK in the hard energy. (b) The example of best-fit X-ray model spectra to the observed spectrum of Itokawa (facets \#10). Same energy peaks are found as in (a). Although Al and S peaks of Itokawa are faint, these $\mathrm{X}$-ray intensities are increasing and decreasing in each facet. Since the backgrounds raise the level of spectral signals, we determined not only best-fit mass fractions but also upper limits for $\mathrm{Al}$ and $\mathrm{S}$.

between face $i$ and XRS-Hayabusa. Table 2 shows average incident and emission angle (average angles for $1800 \mathrm{~s}$ are only indicated) for ten observed areas named facet numbers from \#1 to \#10. The model spectra of fluorescent and scattered X-rays were recalculated for every face and every fitting.

Variation of geometry apparently causes the intensity changes of X-rays. Figure 3 shows the X-ray intensity ratios calculated for fluorescent X-ray emission from multi-faces to mono-plane in the sunlit area for ten facets. In particular, apparent changes for abundance of Fe due to geometry variation are relatively large. Since most of the abundance of sulfur is proportional to that of Troilite $(\mathrm{FeS})$ in asteroids, this geometry correction is important for sulfur estimation.

The size of the polygon face $s_{i}$ is the order of me- 
Table 3. Results of standard sample and Itokawa analysis.

\begin{tabular}{c|cc|ccccc}
\hline & \multicolumn{2}{|c|}{ Standard sample } & \multicolumn{7}{c}{ Itokawa } \\
\hline $\begin{array}{c}\text { Observation } \\
\text { start time }\end{array}$ & \multicolumn{2}{|c|}{$\begin{array}{c}\text { Plasma temp. } \\
\text { Soft }\end{array}$} & Facet & Mg & Al & Si & $\mathrm{S}$ \\
(UTC) & \multicolumn{2}{|c|}{ number } \\
\hline $12: 02: 11$ & $4.56 \pm 0.03$ & $13.72 \pm 0.03$ & 1 & $15.0 \pm 0.1$ & $<2.1(1.8)$ & $18.0 \pm 0.2$ & $<3.2(2.4)$ \\
$12: 39: 52$ & $4.08 \pm 0.06$ & $13.53 \pm 0.04$ & 2 & $15.0 \pm 0.2$ & $<2.1(1.7)$ & $18.0 \pm 0.3$ & $<3.5(2.4)$ \\
$13: 17: 30$ & $3.92 \pm 0.07$ & $13.78 \pm 0.02$ & 3 & $15.0 \pm 0.2$ & $<2.2(1.8)$ & $18.0 \pm 0.2$ & $<2.9(2.1)$ \\
$13: 55: 12$ & $4.28 \pm 0.06$ & $14.35 \pm 0.04$ & 4 & $15.1 \pm 0.3$ & $<1.9(1.5)$ & $18.0 \pm 0.4$ & $<3.4(2.5)$ \\
$14: 32: 53$ & $4.41 \pm 0.03$ & $15.73 \pm 0.02$ & 5 & $15.1 \pm 0.2$ & $<4.8(1.5)$ & $18.0 \pm 0.2$ & $<3.0(2.5)$ \\
$15: 10: 37$ & $4.16 \pm 0.03$ & $15.26 \pm 0.02$ & 6 & $15.2 \pm 0.2$ & $<2.0(1.6)$ & $18.0 \pm 0.3$ & $<2.9(2.2)$ \\
$15: 48: 20$ & $4.50 \pm 0.03$ & $15.52 \pm 0.02$ & 7 & $15.1 \pm 0.1$ & $<3.1(2.0)$ & $18.0 \pm 0.2$ & $<2.7(2.3)$ \\
$16: 26: 04$ & $4.22 \pm 0.11$ & $14.11 \pm 0.07$ & 8 & $15.3 \pm 0.2$ & $<2.9(1.6)$ & $17.8 \pm 0.2$ & $<3.0(2.4)$ \\
$17: 03: 48$ & $4.37 \pm 0.01$ & $14.36 \pm 0.02$ & 9 & $15.2 \pm 0.2$ & $<2.7(2.4)$ & $17.9 \pm 0.2$ & $<2.7(1.9)$ \\
$17: 41: 32$ & $4.36 \pm 0.06$ & $14.88 \pm 0.06$ & 10 & $15.2 \pm 0.2$ & $<1.9(1.6)$ & $17.8 \pm 0.2$ & $<2.9(2.5)$ \\
\hline
\end{tabular}

ters, which is much smaller than the observed area, and the roughness of smaller scale than polygon faces was neglected in this study.

Initial abundance for the fit of X-ray model of Itokawa assumed an average composition of ordinary-chondrites (Jarosewich, 2006; Yanai and Kojima, 1995) in Table 1, including an average of 229 samples of H-chondrites, an average of 256 samples of L-chondrites, and an average of 83 samples of LL-chondrites with each standard deviation.

Free parameters for the fit were elemental mass fractions of $w_{\mathrm{Mg}}, w_{\mathrm{Al}}, w_{\mathrm{Si}}$, and $w_{\mathrm{S}}$. Mass fractions of another elements were fixed in those of average of ordinary chondrites (see Table 1) because observed peaks were faint, such as $\mathrm{Ca}, \mathrm{Fe}$, and ..., in this solar condition. All mass fractions for each element were normalized and recalculated during the fitting iteration as follows, $\sum w_{i}=100 \%$. Also, the ratio of $w_{\mathrm{O}}$ was complemented with oxide ratios, using atomic ratios of $\mathrm{MgO}, \mathrm{Al}_{2} \mathrm{O}_{3}$, and $\mathrm{SiO}_{2}$.

The example of a best-fit X-ray model to the observed spectrum of Itokawa is shown in Fig. 4(b). An unexplained peak, found in the spectra of standard sample, is also found in the spectra of Itokawa. Line peaks of $\mathrm{Mg}$ and $\mathrm{Si}$ are also found, but intensities of these spectra are relatively small, probably due to the condition of the surface, such as roughness. In general, a rough target surface decreases the intensities of emission X-rays. Since the standard sample is the glass plate, X-ray emissions from the standard sample are relatively large compared to those from the rough surface of Itokawa. Additionally, the instrumental background of the asteroid detector was large relative to the solar monitor. The exponential function as spectral backgrounds implies that the instrumental degradation due to space irradiation is progressing. Although spectral backgrounds of these observed spectra are large, these peak heights of Al to Si or S to Si were increasing or decreasing for each spectrum of ten observations, respectively. Therefore, we determined conservatively not only best-fit abundance but also upper limits abundance for $\mathrm{Al}$ and $\mathrm{S}$ in local ten areas.

\section{Results}

We estimated the solar X-ray spectra as the X-ray exciting source and determined the major elemental abundance on the surface of Itokawa for ten areas, and then we care- fully corrected the fluctuation of solar X-rays, geometry variation of local areas, and sensor response function in ten spectra. Table 3 shows the best-fit results, including the solar coronal temperatures for the soft- and hard-energy range, the mass fractions for $\mathrm{Mg}$ and $\mathrm{Si}$, and the upper limits and best-fit mass fractions for $\mathrm{Al}$ and $\mathrm{S}$ for ten areas.

In this observation period, solar temperatures fluctuated between 3.9 and $4.5 \mathrm{MK}$ in the soft-energy range, and between 13.5 and 15.7 MK in the hard-energy range. The maximum temperature was equivalent to the flare levels of C2 (GOES's web site), estimated with Feldman et al.'s equation (6) (Feldman et al., 1996). This flare level is high enough to estimate sulfur abundance within $10 \%$ counting statistical errors. However, space radiation or instrumental background raises the level of spectral signals, and then we were unable to determine the lower limits for $\mathrm{Al}$ and $\mathrm{S}$. Thus, we estimated not only best-fit mass fractions but also upper limits for $\mathrm{Al}$ and $\mathrm{S}$.

The best-fit elemental mass fractions of Itokawa for ten facets are shown in Table 3. The mass fractions for $\mathrm{Mg}$ and $\mathrm{Si}$ are shown with standard errors propagated with modelfit errors, counting statistics errors, and model-fit errors of solar X-ray. The best-fit mass fractions for $\mathrm{Al}$ and $\mathrm{S}$ are shown with upper limits.

The abundances for $\mathrm{Mg}$ and $\mathrm{Si}$ are similar to the average of ordinary chondrites (see Table 1). Although upper limit abundances for $\mathrm{Al}$ and $\mathrm{S}$ are higher than the average abundance of ordinary chondrites, these are within the abundance variation of ordinary chondrites. Also, these abundances are consistent within the errors of the results of our previous report (Okada et al., 2006a). Thus, the major elemental composition on the surface of Itokawa resembles that of ordinary chondrites.

Figure 5(a)-(c) shows elemental ratios for $\mathrm{Mg} / \mathrm{Si}$ vs. $\mathrm{Al} / \mathrm{Si}, \mathrm{Al} / \mathrm{Si}$ vs. $\mathrm{S} / \mathrm{Si}$, and $\mathrm{S} / \mathrm{Si}$ vs. $\mathrm{Mg} / \mathrm{Si}$ of our results together with those of major meteorites (Jarosewich, 2006; Yanai and Kojima, 1995). These meteorites are candidates for S-class asteroids, such as an ordinary chondrites including H-, L-, and LL- chondrites, primitive achondrites including Acapulcoites, Winonaites, and Brachinites, stonyiron meteorites including Pallasites, Mesosiderites, Lodranites, and achondrites including Howardites, Eucrites, Diogenites, Aubrites, Ureilites, and Angrites. The results of 
(a) $\mathrm{Mg} / \mathrm{Si}$ versus $\mathrm{Al} / \mathrm{Si}$ ratios (wt\%)

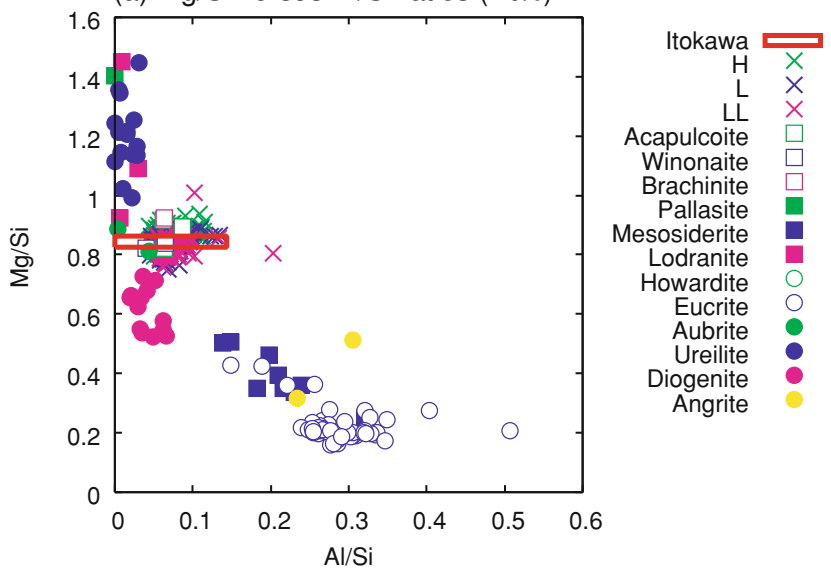

(b) $\mathrm{Al} / \mathrm{Si}$ versus $\mathrm{S} / \mathrm{Si}$ ratios $(\mathrm{wt} \%)$

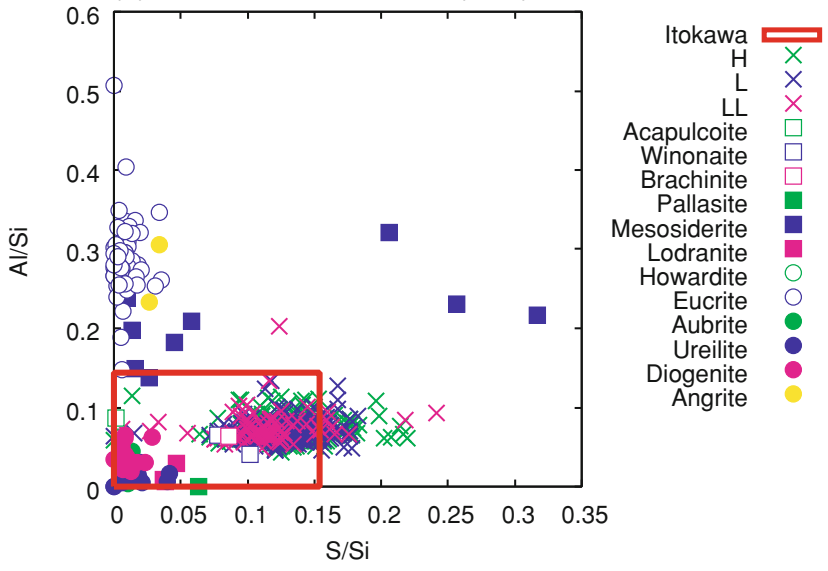

(c) $\mathrm{S} / \mathrm{Si}$ versus $\mathrm{Mg} / \mathrm{Si}$ ratios (wt\%)

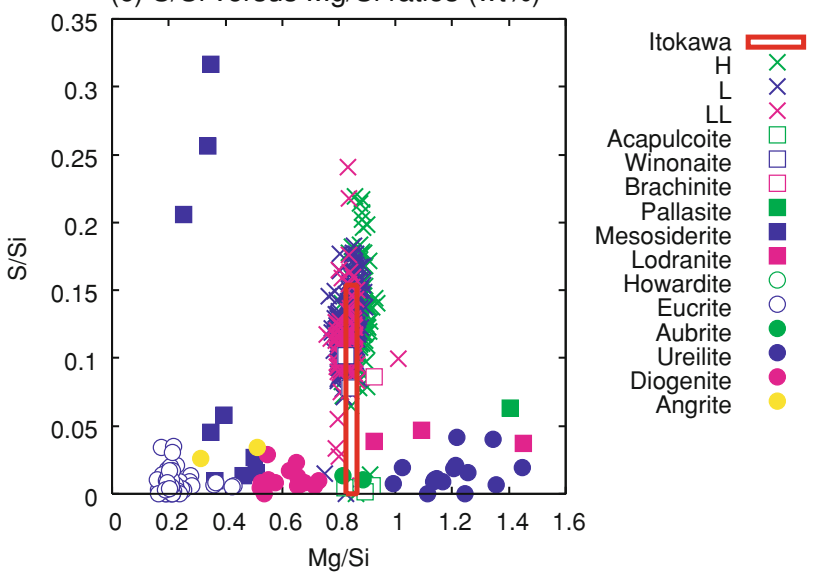

Fig. 5. Color points indicate the mass ratios of $\mathrm{Mg} / \mathrm{Si}, \mathrm{Al} / \mathrm{Si}$, and $\mathrm{S} / \mathrm{Si}$ for meteorites, such as ordinary chondrites including H, L, and LL chondrites, primitive achondrites including Acapulcoites, Winonaites, and Brachinites, stony-iron meteorites including Pallasites, Mesosiderites, Lodranites, and achondrites including Howardites, Eucrites, Aubrites, Ureilites, Diogenites, and Angrites (Jarosewich, 2006; Yanai and Kojima, 1995). Red squares show the upper and lower limits for the observed elemental mass ratios of Itokawa in all facets. Itokawa is best-classified ordinary chondrite or primitive achondrite according to overlapped meteorites analogues in (a), (b), and (c).

upper and lower limits for $\mathrm{Mg} / \mathrm{Si}, \mathrm{Al} / \mathrm{Si}$, and $\mathrm{S} / \mathrm{Si}$ overlap those of meteorite analogues for ordinary chondrites and primitive achondrites. Thus, in terms of major elemental composition, Itokawa is best classified as ordinary chondrite or primitive achondrite in this result.
Here, we calculated a linear-correlation factor (e.g., Bevington and Robinson, 2003) for ten data sets of the ratios for $\mathrm{Mg} / \mathrm{Si}$ vs. $\mathrm{Al} / \mathrm{Si}, \mathrm{Al} / \mathrm{Si}$ vs. $\mathrm{S} / \mathrm{Si}$, and $\mathrm{S} / \mathrm{Si}$ vs. $\mathrm{Mg} / \mathrm{Si}$. These results are $0.03,-0.92$, and 0.09 , respectively. In particular, the correlation factor for $\mathrm{Al} / \mathrm{Si}$ vs. S/Si indicates that there is a negative correlation, despite large errors, and thus it implies that depletion of sulfur is correlated to the enrichment of aluminum, and vice versa.

The abundance of $\mathrm{Mg}$ and $\mathrm{Si}$ appears to be regionally homogeneous, but the best-fit mass fractions and upper limits of $\mathrm{Al}$ and $\mathrm{S}$ vary in local areas. The best-fit mass fractions of sulfur are shown in Fig. 6, plotted on the shape model of Itokawa (Demura et al., 2006). The area with the lowest sulfur is found in facet \#9 accompanied with rich aluminum. This area is close to a large cratered area with many high albedo sites called Arcoona, the top of elongated asteroid.

\section{Discussion}

Our results indicate that the elemental mass fractions for $\mathrm{Mg}, \mathrm{Al}, \mathrm{Si}$, and $\mathrm{S}$ of Itokawa are similar to ordinary chondrite or primitive achondrite in major elemental composition. The regional variation of the abundance of $\mathrm{Mg}$ and $\mathrm{Si}$ are globally homogeneous. The best-fit mass fractions or upper limits of $\mathrm{Al}$ and $\mathrm{S}$ vary in local areas. The correlation of $\mathrm{Al} / \mathrm{Si}$ and $\mathrm{S} / \mathrm{Si}$ is a negative correlation factor of -0.92 for 10 areas. Here, we discuss the actual abundance of sulfur and its regional variation, especially such rich aluminum and lower sulfur in local areas.

In this study, we carefully corrected the fluctuation of solar X-rays, geometry variation of the surface, and sensor response function, and thus we believe that the accuracy of analysis has increased compared to our previous report (Okada et al., 2006a). However, our results do not include some apparent effects to vary the mass fractions, such as a particle size effect and a mineral mixing effect. The particle size effect has been studied by Okada and Kuwada (1997) and Maruyama et al. (2007). This effect will be most apparent when the size of particles on the surface is larger than or as large as the transmission depth of fluorescent X-rays $(<100 \mu \mathrm{m})$, and then this effect causes a decrease in the intensity of fluorescent X-rays in low energy. This effect also depends on the observation phase angle and is not effective at low-phase angles like our observation; therefore, we neglected this effect. The mineral mixing effect has been studied by Nittler et al. (2001) and Akagawa (2003) and cannot be neglected. This effect depends on the volume size of the mineral, and it apparently varies the observed elemental abundance. Since our fluorescent X-ray model in Eq. (1) does not include mineral volume, we must correct this effect. However, since precise mineral volumes on the surface are uncertain, we use the typical one. The typical models of mineral mixing model to homogeneous model for each element, obtained by Nittler et al. (2001) and Akagawa (2003) are shown in Fig. 7. This figure implies that our results of $\mathrm{S} / \mathrm{Si}$ ratio, assuming as homogeneous model, may result in an overestimation by $30 \%$, and thus the true abundance of sulfur on the surface of Itokawa may be smaller than that of our results. If so, sulfur may be globally depleted on the surface of Itokawa. Thus, the abundance of sulfur on the surface of Itokawa is almost equal to or even lower than 


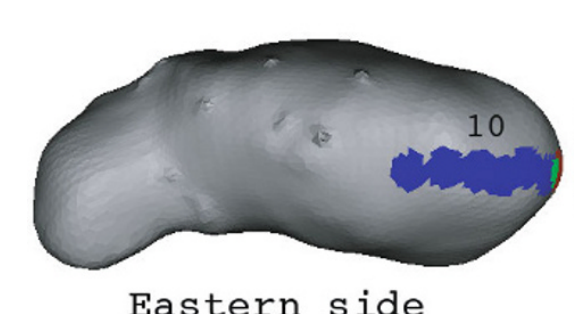

Eastern side

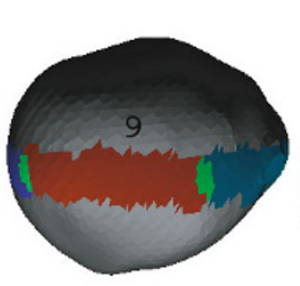

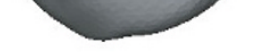

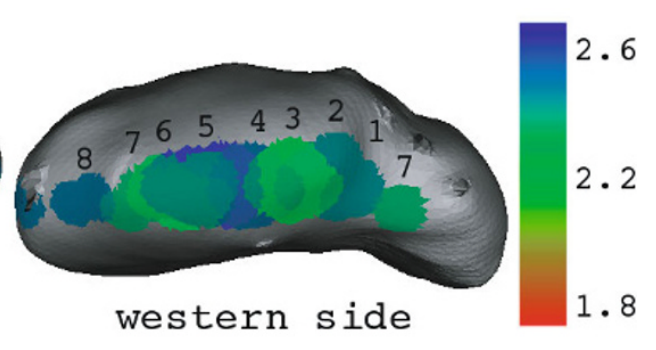

Fig. 6. The best-fit mass fractions of sulfur, plotted on the shape model of Itokawa (Demura et al., 2006). Numbers indicate observation areas called facets. Color map shows the mass fractions of sulfur abundance between 1.8 and 2.6 (wt.\%). The abundances for duplicated observation areas are shown with the average abundance. Although the abundances of Mg and Si are globally homogeneous (see Table 3), the best-fit sulfur abundances vary in local areas. The lowest sulfur area \#9 is close to large cratered area (see figure 3 of Demura et al., 2006).

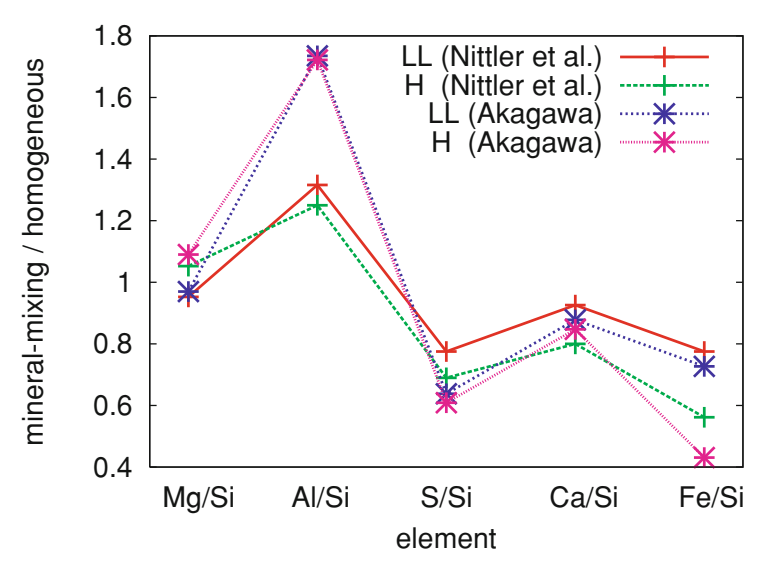

Fig. 7. X-ray fluorescence photon ratios of mineral mixing model to homogeneous model, obtained by Nittler et al. (2001) and Akagawa (2003). The mass ratios of mineral mixing model depend on volume ratios of minerals. Since our fluorescence X-ray model in Eq. (1) does not contain mineral volumes, we must correct the mineral mixing effects. However, the true volumes of minerals on the surface of Itokawa are uncertain. Therefore, we use the typical one as this figure.

that of average abundance of ordinary chondrites, even if we take the upper limit of its abundance.

Since the size of Itokawa is small $\left(535 \times 294 \times 209 \mathrm{~m}^{3}\right)$, probably composed of rubble piles (Fujiwara et al., 2006), partial melting is not effective after the formation of Itokawa. However, fractionation of minerals might have occurred on the parent body of Itokawa. Partial melting may induce the sulfur evaporation on the parent body of Itokawa. It may also induce enrichment in aluminum because aluminum appears in the first melt of chondritic material at sulfur evaporation temperature. Portions of partial melting features have been found on Itokawa in about a $2-\mu \mathrm{m}$ absorption band of NIRS-Hayabusa and in the Earth-based observations (Abell et al., 2006). This result indicates that a portion of Itokawa is more olivine-rich than primitive ordinary chondrites. Thus, our results of the rich aluminum and lower sulfur features may support the idea that partial melting events occurred on the parent body of Itokawa.

The area with the lowest best-fit sulfur area is facet \#9, close to the large cratered area. This area may have experienced a heating process due to impacts, and thus the sulfur was vaporized. Aluminum enrichment is also found in facet \#9. One possible cause is a meteoritic impact of Al- enriched material. Although a high-speed impact explodes and ejects the surface objects, a low-speed impact leaves its portion on the surface. Not only aluminum-rich but also chondritic materials, such as carbonaceous chondrites, are present. The black boulder on the surface of Itokawa at a longitude of $0^{\circ}$ (see figure 1 of Saito et al., 2006) may be a remnant of a low-speed impactor such as C-class asteroids. Thus, our results of the aluminum enrichment and sulfur depletion may support the idea that an aluminum-rich impact occurred on the surface of Itokawa.

The geometric albedo of Itokawa ( $\sim 30 \%$; see Lederer et al., 2005) is brighter than that of 433 Eros ( 25\%; see Li et al., 2004), and also color variation on the surface of Itokawa was found with AMICA-Hayabusa (Ishiguro et al., 2006). Michel and Yoshikawa (2005) have computed that the probability of Itokawa colliding with the Earth is high within $10^{6}$ years, so that a planet encounter with Itokawa might occur. Sasaki et al. (2006) have suggested that the resurfacing process on Itokawa may be caused by seismic destruction due to impacts or tidal stress as a result of planet encounters, and thus the portion of exposed material on the surface may be little altered materials by space weathering. Killen (2003) and Kracher and Sears (2005) have suggested that it is easy to remove the sulfur on the asteroid surface by space weathering in the uppermost layer of 10-100 $\mu \mathrm{m}$ per $10^{6}$ years, and thus the sulfur abundance on the surface may vary between little and highly altered areas. Since our result did not indicate a global depletion of sulfur, in spite of large errors, the abundance of sulfur may be fresh in local areas due to the resurfacing process compared to highly altered materials by space weathering. Thus, our results of sulfur variation may reflect the heterogeneity of the surface, altered by space weathering.

\section{Conclusion}

We determined the elemental abundance for $\mathrm{Mg}$ and $\mathrm{Si}$, and best-fit and upper limit abundances for $\mathrm{Al}$ and $\mathrm{S}$ in the ten equatorial areas of Itokawa. In particular, we carefully corrected the fluctuation of solar X-rays, variation of surface geometry, and sensor response function in this analysis, and thus we believe that the results will have more accuracy than our previous report (Okada et al., 2006a). In the results of this study, the abundances of $\mathrm{Mg}$ and $\mathrm{Si}$ are near the average abundance of ordinary chondrites and globally homogeneous in ten areas. Although upper limit abundances for 
$\mathrm{Al}$ and $\mathrm{S}$ exceed the average abundance of ordinary chondrites, these are within the variation of ordinary chondrites. The results of upper and lower limits for $\mathrm{Mg} / \mathrm{Si}, \mathrm{Al} / \mathrm{Si}$, and $\mathrm{S} / \mathrm{Si}$ overlap that of meteorite analogues for those of ordinary chondrites or primitive achondrites. Thus, in terms of major elemental composition, Itokawa is best classified as an ordinary chondrite or primitive achondrite.

Our X-ray models do not include the mineral mixing effects, and thus the true abundance of sulfur may be lower than our estimation. If so, sulfur is globally depleted on the surface of Itokawa. Hence, we conclude that the abundance of sulfur on the surface of Itokawa is almost equal to or even lower than that of average abundance of ordinary chondrites.

Although the abundances for $\mathrm{Mg}$ and $\mathrm{Si}$ on the surface of Itokawa are globally homogeneous, best-fit and upper limits abundances of $\mathrm{Al}$ and $\mathrm{S}$ vary in local areas. There are negative correlations in $\mathrm{Al} / \mathrm{Si}$ vs. $\mathrm{S} / \mathrm{Si}(-0.92)$ for ten facets. In particular, a rich aluminum and lower sulfur area (in terms of best-fit abundance) is found in facet \#9, called Arcoona, close to a cratered area. Therefore, the rich aluminum and lower sulfur features may support that events of partial melting on the parent body of Itokawa took place or aluminum-rich material impacted on the surface of Itokawa.

In some areas, the geometric albedo of Itokawa is brighter and varies in color. Little altered, fresh material may be exposed in these portions of the surface. The sulfur abundance on the surface appear to vary between areas highly and little altered by space weathering. Thus, the sulfur regional variation in our result may reflect the heterogeneity of the surface altered by space weathering.

Acknowledgments. We thank A. Hagermann and S. Tanaka for helpful comments and suggestions. We also thank all the member of the Hayabusa project.

\section{References}

Abe, M., Y. Takagi, K. Kitazato, S. Abe, T. Hiroi, F. Vilas, B. E. Clark, P. A. Abell, S. M. Lederer, K. S. Jarvis, T. Nimura, Y. Ueda, and A. Fujiwara, Near-Infrared Spectral Results of Asteroid Itokawa from the Hayabusa Spacecraft, Science, 312, 1334-1338, 2006.

Abell, P. A., F. Vilas, K. S. Jarvis, M. J. Gaffey, and M. S. Kelley, Mineralogical Composition of (25143) Itokawa 1998 SF36 from Visible and Near-Infrared Reflectance Spectroscopy: Evidence for Partial Melting, LPI, 37, 1513A, 2006.

Akagawa, K., Master thesis of The University of Tokyo, 2003 (in Japanese).

Anders, E. and N. Grevesse, Abundances of the elements-Meteoritic and solar, Geochim. Cosmochim. Acta, 53, 197-214, 1989.

Arai, T., Master thesis of Tokyo Institute of Technology, 2003.

Arnaud, K., B. Dorman, and C. Gordon, Xspec An X-ray Spectral Fitting Package User's Guide for version 12.2, HEASARC, Exploration of the Universe Division, NASA/GSFC, 2005.

Bearden, J. A., X-Ray Wavelengths, Rev. Mod. Phys., 39, 78-124, 1967.

Bevington, P. R. and D. K. Robinson, Testing The Fit, in Data Reduction and Error Analysis for the Physical Sciences, $3 r d$ ed., 320 pp, McGrawHill, New York, 2003.

Binzel, R. P., A. S. Rivkin, S. J. Bus, J. M. Sunshine, and T. H. Burbine, MUSES-C target asteroid (25143) 1998 SF36: A reddened ordinary chondrite, Meteor. Planet. Sci., 36(8), 1167-1172, 2001.

Chantler, C. T., K. Olsen, R. A. Dragoset, J. Chang, A. R. Kishore, S. A. Kotochigova, and D. S. Zucker, 2005, X-Ray Form Factor, Attenuation and Scattering Tables (version 2.1). [Online] Available: http://physics.nist.gov/ffast [2006, October 25]. National Institute of Standards and Technology, Gaithersburg, MD. Originally published as
Chantler, C. T., J. Phys. Chem. Ref. Data, 29(4), 597-1048, 2000; and Chantler, C. T., J. Phys. Chem. Ref. Data, 24, 71-643, 1995.

Clark, P. E. and J. I. Trombka, Remote X-ray spectrometry for NEAR and Future missions: Modeling and analyzing X-ray production from source to surface, J. Geophys. Res., 102(E7), 16361-16384, 1997.

Demura, H., S. Kobayashi, E. Nemoto, N. Matsumoto, M. Furuya, A. Yukishita, N. Muranaka, H. Morita, K. Shirakawa, M. Maruya, H. Ohyama, M. Uo, T. Kubota, T. Hashimoto, J. Kawaguchi, A. Fujiwara, J. Saito, S. Sasaki, H. Miyamoto, and N. Hirata, Pole and Global Shape of 25143 Itokawa, Science, 312, 1347-1349, 2006.

Feldman, U., G. A. Doschek, W. E. Behring, and K. J. H. Phillips, Electron Temperature, Emission Measure, and X-Ray Flux in A2 to X2 X-Ray Class Solar Flares, ApJ, 460, 1034-1041, 1996.

Fujiwara, A., M. Abe, Y. Takagi, K. Kitazato, S. Abe, T. Hiroi, F. Vilas, B. E. Clark, P. A. Abell, S. M. Lederer, K. S. Jarvis, T. Nimura, Y. Ueda, and A. Fujiwara, Near-Infrared Spectral Results of Asteroid Itokawa from the Hayabusa Spacecraft, Science, 312, 1334-1338, 2006.

GOES's web site, http://www.sec.noaa.gov/.

Hayabusa's web site, http://hayabusa.sci.isas.jaxa.jp/.

HEASARC's web site, http://heasarc.gsfc.nasa.gov/docs/asca/gis_night earth/gis_night_earth.html.

Ishiguro, M. I., T. H. Hiroi, D. J. T. Tholen, A. Y. Yamamoto, S. S. Sasaki F. Y. Yoshida, B. E. Clark, R. N. Nakamura, and J. S. Saito, Detection of a Large Variation in the Degree of Space Weathering on the Surface of Itokawa by Hayabusa/AMICA Observations, LPI, 37, 1533I, 2006.

Jarosewich, E., Chemical analyses of meteorites at the Smithsonian Institution: An update, $M \& P S$, 41(9), 1271-1419, 2006.

Jenkins, R., R. W. Gould, and D. Gedcke, The interaction of X-rays with Matter, in Quantitative X-ray Spectrometry 2 nd Ed., edited by Brame, E. G, Jr., 504 pp., Marcel Dekker, Inc, New York·Basel·Hong Kong, 1995.

Killen, R. M., Depletion of sulfur on the surface of asteroids and the moon, $M \& P S$, 38(3), 383-388, 2003.

Knoll, G. F., Counting Statistics and Error Prediction, in Radiation detection and measurement $3 r d$ ed., 802 pp., Wiley, New York, 2000.

Kracher, A. and D. W. G. Sears, Space weathering and the low sulfur abundance of Eros, Icarus, 174(1), 36-45, 2005.

Krause, M. O., Atomic radiative and radiationless yields for $\mathrm{K}$ and $\mathrm{L}$ shells, J. Phys. Chem. Ref. Data, 8, 307-327, 1979.

Lederer, S. M., D. L. Domingue, F. Vilas, M. Abe, T. L. Farnham, K. S. Jarvis, S. C. Lowry, Y. Ohba, P. R. Weissman, L. M. French, H. Fukai, S. Hasegawa, M. Ishiguro, S. M. Larson, and Y. Takagi, Physical characteristics of Hayabusa target Asteroid 25143 Itokawa, Icarus, 173(1), $153-165,2005$.

Li, J., M. F. A'Hearn, and L. A. McFadden, Photometric Studies of Eros from NEAR Data, LPI, 35, 2080, 2004.

Maruyama, Y., K. Ogawa, T. Okada, and M. Kato, Particle Size Effect in XRay Fluorescence and Its Implication to Planetary XRF Spectroscopy, LPI, 1338, 2007.

Maslen, E. N., A. G. Fox, and M. A. O'Keefe, X-ray scattering in International Table for Crystallography, Vol. C 3rd Ed., edited by Prince, E., 1000+xxxii pp., NIST Center for Neutron Research, National Institutes of Standards and Technology, Gaithersburg, USA, 2004.

Masuda, E., Master thesis of Tokyo Institute of Technology, 2002.

Mewe, R., E. H. B. M. Gronenschild, and G. H. J. van den Oord, Calculated X-radiation from optically thin plasmas. V, A\&AS, 62, 197-254, 1985.

Michel, P. and M. Yoshikawa, Earth impact probability of the Asteroid (25143) Itokawa to be sampled by the spacecraft Hayabusa, Icarus, 179(2), 291-296, 2005.

Nittler, L. R., R. D. Starr, L. Lim, T. J. McCoy, T. H. Burbine, R. C. Reedy, J. I. Trombka, P. Gorenstein, S. W. Squyres, W. V. Boynton, T. P. McClanahan, J. S. Bhangoo, P. E. Clark, M. E. Murphy, and R. Killen, $\mathrm{X}$-ray fluorescence measurements of the surface elemental composition of asteroid 433 Eros, $M \& P S$, 36(12), 1673-1695, 2001.

Okada, T. and Y. Kuwada, Effect of surface roughness on X-ray fluorescence emission from planetary surfaces, $L P I, \mathbf{2 8}, 1997$.

Okada, T., M. Kato, A. Fujimura, H. Tsunemi, and S. Kitamoto, X-ray fluorescence spectrometer onboard Muses-C, AdSpR, 25(2), 345-348, 2000.

Okada, T., K. Shirai, Y. Yamamoto, T. Arai, K. Ogawa, K. Hosono, and M. Kato, X-ray Fluorescence Spectrometry of Asteroid Itokawa by Hayabusa, Science, 312, 1338-1341, 2006a.

Okada, T., K. Shirai, Y. Yamamoto, T. Arai, K. Ogawa, K. Hosono, and M. Kato, Instrumentation and observations of the X-ray spectrometer onboard Hayabusa, in Advances in Geosciences, 3: Planetary Science (PS), edited by Bhardwaj, A., World Scientific, Singapore, 231-240, 2006b. 
Press, W. H., S. A. Teukolsky, W. T. Vetterling, and B. P. Flannery, Minimization or Maximization of Functions in Numerical Recipes in $\mathrm{C}++$ 2nd Ed., edited by Press, W. H., Cambridge University Press, Cambridge, 1002 pp., 2002a.

Press, W. H., S. A. Teukolsky, W. T. Vetterling, and B. P. Flannery, Modeling of Data, in Numerical Recipes in $C++2 n d E d$., edited by Press, W. H., Cambridge University Press, Cambridge, 1002 pp., $2002 \mathrm{~b}$.

Saito, J., H. Miyamoto, R. Nakamura, M. Ishiguro, T. Michikami, A. M. Nakamura, H. Demura, S. Sasaki, N. Hirata, C. Honda, A. Yamamoto, Y. Yokota, T. Fuse, F. Yoshida, D. J. Tholen, R. W. Gaskell, T. Hashimoto, T. Kubota, Y. Higuchi, T. Nakamura, P. Smith, K. Hiraoka, T. Honda, S. Kobayashi, M. Furuya, N. Matsumoto, E. Nemoto, A. Yukishita, K. Kitazato, B. Dermawan, A. Sogame, J. Terazono, C. Shinohara, and H. Akiyama, Detailed Images of Asteroid 25143 Itokawa from Hayabusa, Science, 312, 1341-1344, 2006.

Sasaki, S., J. Saito, M. Ishiguro, N. Hirata, H. Miyamoto, H. Demura, T. Hashimoto, Y. Higuchi, K. Hiraoka, C. Honda, T. Honda, K. Kitazato, T. Kubota, T. Michikami, A. M. Nakamura, R. Nakamura, T. Nakamura, P. Smith, J. Terazono, D. J. Tholen, A. Yamamoto, Y. Yokota, H. Akiyama, B. Dermawan, T. Fuse, C. Shinohara, A. Sogame, F. Yoshida, and AM-
ICA Team, Observations of 25143 Itokawa by the Asteroid Multiband Imaging Camera (AMICA) of Hayabusa: Morphology of Brighter and Darker Areas, LPI, 37, 1325, 2006.

Thomson, J. J., Conduction of Electricity Through Gases, 325 pp., Cambridge Univ. Press, London, 1906.

Trombka, J. I., S. W. Squyres, J. Brckner, W. V. Boynton, R. C. Reedy, T. J. McCoy, P. Gorenstein, L. G. Evans, J. R. Arnold, R. D. Starr, L. R. Nittler, M. E. Murphy, I. Mikheeva, R. L. McNutt, T. P. McClanahan, E. McCartney, J. O. Goldsten, R. E. Gold, S. R. Floyd, P. E. Clark, T. H. Burbine, J. S. Bhangoo, S. H. Bailey, and M. Petaev, The Elemental Composition of Asteroid 433 Eros: Results of the NEAR-Shoemaker X-ray Spectrometer, Science, 289, 2101-2105, 2000.

Yamamoto, Y., Doctor thesis of The University of Tokyo, 2002 (in Japanese).

Yanai, K. and H. Kojima, Catalogue of the Antarctic Meteorites, 230 pp., National Institute of Polar Research, Tokyo, 1995.

T. Arai (e-mail: arai@planeta.sci.isas.jaxa.jp), T. Okada, Y. Yamamoto, K. Ogawa, K. Shirai, and M. Kato 